\title{
Влияние двух- и трехслойных просветляющих покрытий на формирование фототоков в многопереходных солнечных элементах на основе $\mathrm{A}^{\text {III }} \mathrm{B}^{\mathrm{V}}$
}

\author{
(C) С.Б. Мусалинов ${ }^{1}$, А.П. Анзулевич ${ }^{1}$, И.В. Бычков ${ }^{1}$, А.С. Гудовских ${ }^{2}$, М.З. Шварц ${ }^{3 థ}$ \\ 1 Челябинский государственный университет, \\ 454021 Челябинск, Россия \\ ${ }^{2}$ Санкт-Петербургский национальный исследовательский Академический университет Российской академии наук, \\ 194021 Санкт-Петербург, Россия \\ ${ }^{3}$ Физико-технический институт им. А.Ф. Иоффе Российской академии наук, \\ 194021 Санкт-Петербург, Россия \\ ๆ E-mail: shvarts@scell.ioffe.ru
}

(Получена 20 июня 2016 г. Принята к печати 29 июня 2016 г.)

\begin{abstract}
Представлены результаты моделирования методом матриц переноса двухслойного, $\mathrm{TiO}_{2} / \mathrm{SiO}_{2}$, и трехслойного, $\mathrm{TiO}_{2} / \mathrm{Si}_{3} \mathrm{~N}_{4} / \mathrm{SiO}_{2}$, просветляющих покрытий для многопереходных гетероструктурных солнечных элементов $\mathrm{InGaP} / \mathrm{GaAs} / \mathrm{Ge}$. Экспериментально получено и оптимизировано двухслойное просветляющее покрытие $\mathrm{TiO}_{2} / \mathrm{SiO}_{2}$. С использованием экспериментальных спектральных зависимостей квантового выхода фотоответа солнечного элемента $\mathrm{InGaP} / \mathrm{GaAs} / \mathrm{Ge}$ и оптических характеристик просветляющих покрытий, полученных при моделировании, определены значения плотностей фототоков отдельных каскадов трехпереходного солнечного элемента для условий облучения AM1.5D $\left(1000 \mathrm{BT} / \mathrm{M}^{2}\right)$, а также для случая отсутствия потерь на отражение. В рамках моделирования показано, что оптимизированное трехслойное просветляющее покрытие $\mathrm{TiO}_{2} / \mathrm{Si}_{3} \mathrm{~N}_{4} / \mathrm{SiO}_{2}$ дает выигрыш в величине плотности фототока на $2.3 \mathrm{~mA} / \mathrm{cm}^{2}$ (AM1.5D) для Ge-субэлемента по сравнению с рассматриваемым вариантом двухслойного просветляющего покрытия $\mathrm{TiO}_{2} / \mathrm{SiO}_{2}$, обеспечивая тем самым рост коэффициента заполнения вольт-амперной характеристики и выходной электрической мощности многопереходного солнечного элемента.
\end{abstract}

DOI: 10.21883/FTP.2017.01.44001.8355

\section{1. Введение}

Повышение эффективности фотоэлектрического преобразования солнечного излучения является одной из главных задач при создании новых типов солнечных элементов (СЭ). Для широко используемых в настоящее время в космической и наземной солнечной энергетике трехпереходных СЭ [1], а также активно разрабатываемых четырех- и шести-переходных элементов [2-4] создание эффективного просветляющего покрытия (ПП) с целью минимизации оптических потерь, определяемых отражением, является одним из важных этапов при решении задачи повышения кпд СЭ.

Если рассматривать СЭ с числом фотоактивных $p-n$-переходов от трех и более $[2,5]$, спектральная чувствительность которых охватывает диапазон длин волн от $\sim 300$ до $\sim 1800$ нм [6], то просветляющих свойств двухслойного диэлектрического покрытия может быть недостаточно для минимизации отражения во всем указанном оптическом диапазоне. Поэтому актуальной является разработка и создание трехслойных ПП для многопереходных СЭ.

В данной работе представлены результаты моделирования методом матриц переноса оптических характеристик двухслойного, $\mathrm{TiO}_{2} / \mathrm{SiO}_{2}$, и трехслойного, $\mathrm{TiO}_{2} / \mathrm{Si}_{3} \mathrm{~N}_{4} / \mathrm{SiO}_{2}$, ПП для $\mathrm{C} \ni \mathrm{InGaP} / \mathrm{GaAs} / \mathrm{Ge}$, а также проведено сравнение результатов расчетов с данными экспериментов для оптимизированных двухслойных ПП $\mathrm{TiO}_{2} / \mathrm{SiO}_{2}$.

\section{2. Моделирование отражения электромагнитной волны слоистыми структурами}

Известно, что для случая нормального падения электромагнитной волны на двухслойную структуру „подложка-просветляющая пленка“ с показателями преломления компонентов соответственно $n_{s}$ и $n_{1}$ условие минимума отражения определяется соотношениями

$$
\begin{gathered}
n_{1}^{2}=n_{0} n_{s}, \\
n_{1} d=\frac{\lambda}{4},
\end{gathered}
$$

где $n_{0}-$ показатель преломления воздуха, $d-$ толщина пленки, $\lambda$ - длина волны света в пленке $\left(n_{1}, n_{s}-\right.$ вещественные величины, $\left.n_{s}>n_{1}\right)$.

Однако представленное математическое описание процесса минимизации отражения не может быть адекватным в случае многослойной структуры $\mathrm{InGaP} /$ $\mathrm{GaAs} / \mathrm{Ge}$, для которой и будет проводиться оптимизация ПП. Во-первых, в случае многопереходного $\mathrm{C} \ni \mathrm{InGaP} / \mathrm{GaAs} / \mathrm{Ge}$ требуемая минимизация отражения в широком спектральном диапазоне не представляется возможной при использовании однослойного ПП. Во-вторых, тонкие слои многослойной структуры СЭ, такие как, например, широкозонное окно AlInP, в силу свойственных им показателей преломления играют роль 
дополнительных интерференционных слоев, влияя на результирующие свойства ПП. Поэтому, очевидно, необходимы адекватные модели, описывающие распространение электромагнитной волны в многослойных комбинированных средах, состоящих их диэлектрических пленок и полупроводниковых наноразмерных слоев. Одним из возможных методов анализа распространения света в многослойной среде является метод матриц переноса [7]. Вся многослойная структура СЭ, включая ПП, может рассматриваться как слоистая среда с постоянными значениями показателя преломления $n$ для каждого слоя.

Рассмотрим нормальное падение электромагнитной ТЕ-волны на поверхность СЭ с ПП. Характеристическую матрицу среды можно записать в виде

$$
M(z)=\left[\begin{array}{cc}
\cos \left(k_{0} n z\right) & -i \frac{\sin \left(k_{0} n z\right)}{p} \\
-i p \sin \left(k_{0} n z\right) & \cos \left(k_{0} n z\right)
\end{array}\right],
$$

где $k_{0}$ - волновое число в вакууме, $n-$ показатель преломления, $p=\sqrt{\varepsilon / \mu}, \varepsilon, \mu-$ диэлектрическая и магнитная проницаемости слоя, в нашем случае $\mu=1$.

В случае $\mathrm{C} Э \mathrm{InGaP} / \mathrm{GaAs} / \mathrm{Ge}$ характеристическая матрица слоистой среды, состоящей из $N$ слоев, будет определяться как

$$
M=\prod_{j=1}^{N} M_{j} .
$$

При рассмотрении ТЕ-волны, падающей на слоистую среду общей толщиной от $z=0$ до $z=z_{1}$, ограниченную с обеих сторон однородными оптическими полубесконечными областями, выражение для коэффициента отражения примет вид

$$
r=\frac{R}{A}=\frac{\left(m_{11}+m_{12} p_{l}\right) p_{1}-\left(m_{21}+m_{22} p_{l}\right)}{\left(m_{11}+m_{12} p_{l}\right) p_{1}+\left(m_{11}+m_{12} p_{l}\right)},
$$

где $A$ и $R$ - амплитуды электрических векторов падающей и отраженной волн, $m_{i j}$ - элементы характеристической матрицы слоистой среды при $z=z_{1}$, $p_{1}=\sqrt{\varepsilon_{1} / \mu_{1}}, p_{1}=\sqrt{\varepsilon_{l} / \mu_{l}}, \varepsilon_{1}, \mu_{1}$ и $\varepsilon_{l}, \mu_{l}$ - диэлектрические и магнитные проницаемости первой и последней сред.

Используя формулы (1)-(3), можно рассчитать коэффициент отражения ТЕ-волны при заданных толщинах слоев, составляющих ПП.

При расчете ПП на основе пленок $\mathrm{TiO}_{2}, \mathrm{Si}_{3} \mathrm{~N}_{4}, \mathrm{SiO}_{2}$ полагалось, что исследуемые пленки оптически прозрачны, а также что показатели преломления материалов постоянны во всем рассматриваемом диапазоне длин волн и составляют $2.45,2.0,1.45$ соответственно [8-10]. Похожий подход был использован при моделировании отражения в работе [11].

Моделирование проводилось для структуры СЭ InGaP/GaAs/Ge c окном AlInP толщиной 0.03 мкм (показатель преломления 3.0). Из формул (1)-(3) видно, что коэффициент отражения в неявном виде зависит от диэлектрической проницаемости $(p=\sqrt{\varepsilon / \mu})$, которая, в

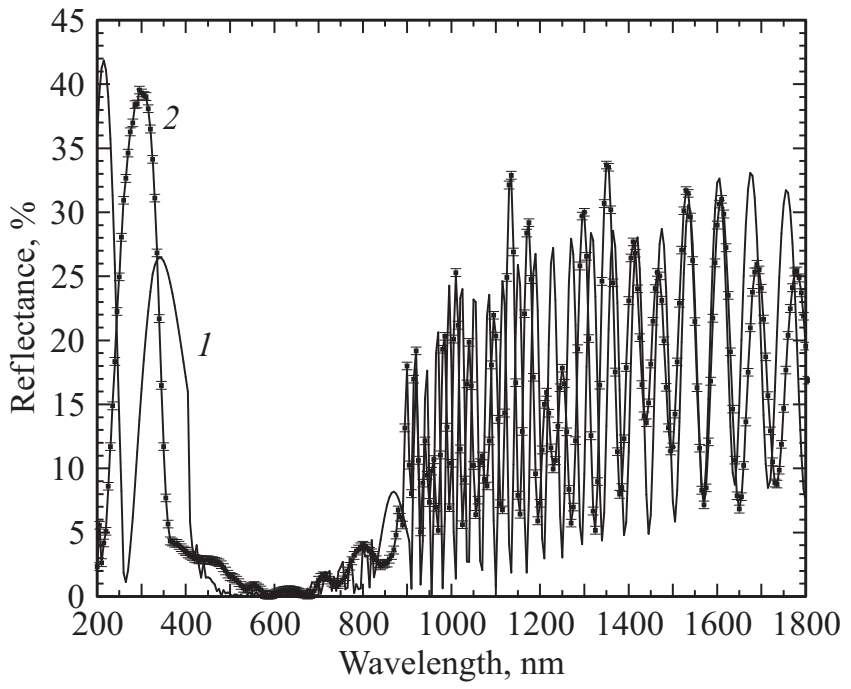

Рис. 1. Спектральные зависимости коэффициента отражения двухслойного ПП $\mathrm{TiO}_{2} / \mathrm{SiO}_{2}: 1-$ эксперимент (толщины слоев 28, 99 нм); 2 - моделирование (толщины слоев 35, 95 нм). 2 - показаны „усы“ погрешности.

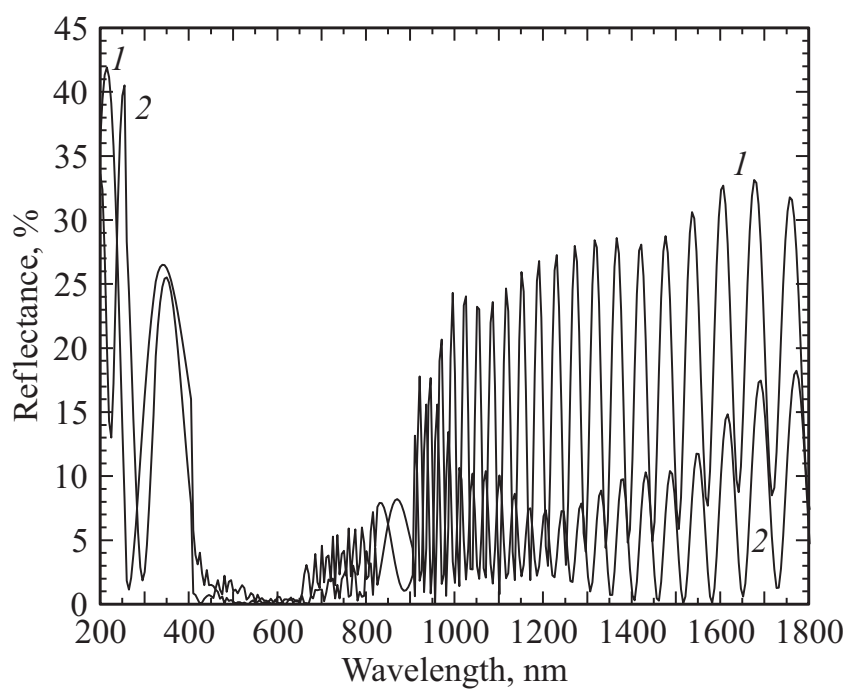

Рис. 2. Спектральные зависимости коэффициента отражения для двух- и трехслойных ПП: $1-\mathrm{TiO}_{2} / \mathrm{SiO}_{2}(35,95$ нм), $2-$ $\mathrm{TiO}_{2} / \mathrm{Si}_{3} \mathrm{~N}_{4} / \mathrm{SiO}_{2}(40,80,100 \mathrm{HM})$.

свою очередь, зависит от длины волны излучения. Поэтому при моделировании спектральных зависимостей коэффициента отражения учитывались как толщины слоев, входящих в состав InGaP-, GaAs-, Ge-субэлементов, так и полученные аналитические зависимости мнимых и вещественных частей диэлектрических проницаемостей от длины волны $\lambda$ для этих слоев [12-14].

Используя эти зависимости в формулах (1)-(3) и варьируя толщины слоев ПП, находили их комбинации, обеспечивающие минимальное отражение в диапазоне спектральной чувствительности многопереходного СЭ. Критерием оптимальной комбинации толщин ПП, соот- 
ветствующей минимальному отражению в спектральном диапазоне $\lambda=300-1800$ нм, являлся минимум площади под получаемой спектральной зависимостью коэффициента отражения.

На рис. 1 представлен смоделированный спектр отражения образца гетероструктуры с двухслойным ПП $\mathrm{TiO}_{2} / \mathrm{SiO}_{2}$, толщины слоев которого составляют 35 , 95 нм, и экспериментально измеренный спектр отражения оптимизированного двухслойного ПП $\mathrm{TiO}_{2} / \mathrm{SiO}_{2}$ с толщинами слоев 28, 99 нм соответственно. Получено достаточно хорошее согласие смоделированного спектра отражения с практически полученным в эксперименте, что свидетельствует об адекватности применяемой расчетной модели.

Усложнение ПП при переходе к трехслойной структуpe $\mathrm{TiO}_{2} / \mathrm{Si}_{3} \mathrm{~N}_{4} / \mathrm{SiO}_{2}(40,80,100 \mathrm{Hм}$ ) (рис. 2) позволяет существенно снизить отражение в инфракрасном диапазоне (1000-1800 нм) по сравнению с применяемой двухслойной комбинацией $\mathrm{TiO}_{2} / \mathrm{SiO}_{2}(28,99$ нм$)$.

\section{3. Нанесение тонких пленок}

Слои $\mathrm{TiO}_{2}$ и $\mathrm{SiO}_{2}$ наносились методом реактивного магнетронного распыления на установке Balzers BAS 450 PM, в которой подложки размещаются на боковой грани вращающегося барабана - подложкодержателя емкостью до шести двухдюймовых подложек (рис. 3). Выбранный метод нанесения ПП обеспечивает равномерное распределение напыляемых слоев при достаточно точном контроле их толщины.

Для слоев $\mathrm{TiO}_{2}$ использовался метод распыления мишени Ті на постоянном токе в среде кислорода. После откачки напылительной камеры до предельного давления $4 \cdot 10^{-4}$ Па подложки прогревались до температуры $60^{\circ} \mathrm{C}$. Для поджига плазмы перед началом процесса распыления в рабочую камеру напускался $\mathrm{Ar}$ до давления 0.5 Па, затем поток $\mathrm{Ar}$ уменьшался до 0 с одновременным увеличением потока кислорода до $98.2 \mathrm{~cm}^{3} / \mathrm{c}$, при этом давление кислорода в камере было равно 0.41 Па. Мощность генератора постоянного тока устанавливалась равной 2 кВт. В течение 5 мин происходила тренировка мишени на заслонку. В момент начала процесса нанесения слоя $\mathrm{TiO}_{2}$ заслонка, закрывающая подложку от магнетрона, открывалась.

Слои $\mathrm{SiO}_{2}$ наносились методом реактивного высокочастотного (ВЧ) магнетронного распыления мишени $\mathrm{SiO}_{2}$ в среде аргона с добавлением кислорода. Как и в предыдущем случае, по достижении предельного давления $4 \cdot 10^{-4}$ Па осуществлялся прогрев подложек до температуры $60^{\circ} \mathrm{C}$. Перед началом процесса распыления устанавливались поток $\operatorname{Ar} 113 \mathrm{~cm}^{3} / \mathrm{c}$ и поток кислорода $18 \mathrm{~cm}^{3} / \mathrm{c}$, при этом давление кислорода в камере составляло 0.64 Па. Мощность ВЧ генератора устанавливалась равной 1.75 Вт. Перед напылительным процессом проводилась тренировка мишени на заслонку в течение 5-10 мин.

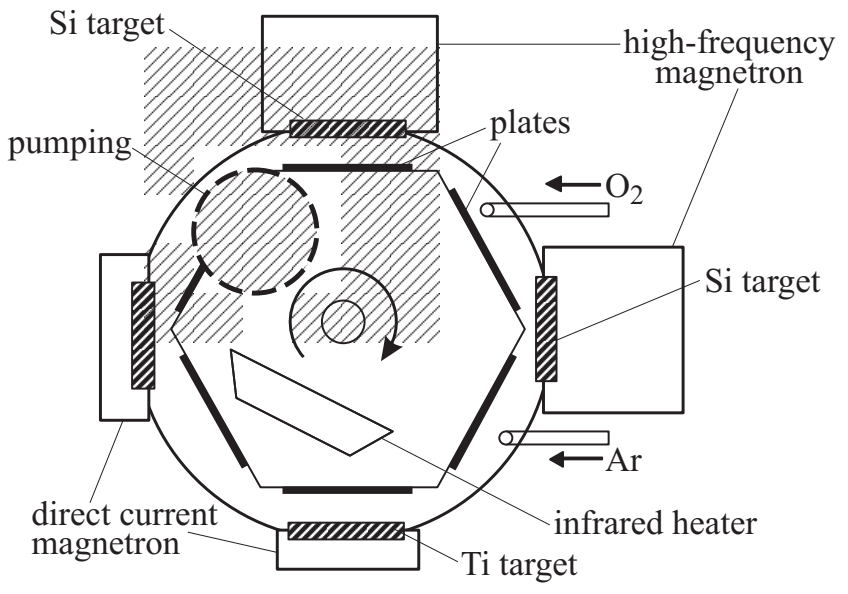

Рис. 3. Схема установки Balzers BAS 450 PM.

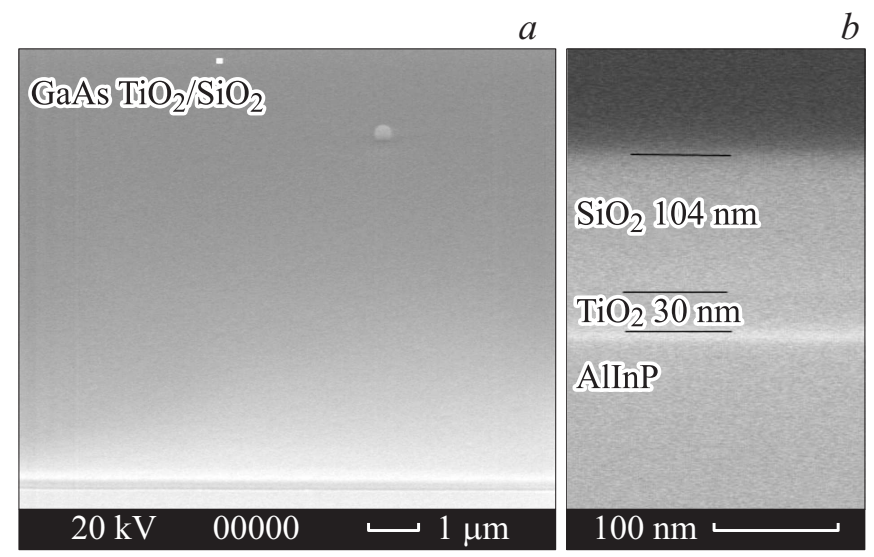

Рис. 4. Полученные методом сканирующей электронной микроскопии фотографии поверхности просветляющего покрытия $\mathrm{TiO}_{2} / \mathrm{SiO}_{2}$, нанесенного на поверхность подложки $\mathrm{GaAs}(a)$, протравленную поверхность широкозонного окна AlInP к слою GaInP $(b)$.

Слои $\mathrm{TiO}_{2}$ и $\mathrm{SiO}_{2}$, осажденные на подложки из GaAs или предметного стекла, использовались для контроля их оптических параметров и оценки скорости роста. Толщина и показатель преломления пленок определялись на эллипсометре „Rudolf research“ (длина волны излучения 632.8 нм). Дополнительно значение толщины проверялось с помощью профилометра Dectak 3030. В слоях $\mathrm{TiO}_{2}$ показатель преломления находился в пределах $2.4-2.5$, а скорость роста составляла $\sim 9$ нм за один оборот барабана для выбранного режима осаждения. Для слоев $\mathrm{SiO}_{2}$ показатель преломления составил $\sim 1.45$, а скорость роста $\sim 30$ нм за один оборот.

На рис. 4 представлены фотографии, полученные методом сканирующей электронной спектроскопии для изготовленных структур (показаны верхние слои). Слой ПП имеет практически зеркальную поверхность. Дополнительно для точного определения толщин слоев использовались данные растровой электронной микроскопии сколов структур. 
Таблица 1. Пределы абсолютной погрешности измерения коэффициента отражения для спектрофотометра Perkin Elmer lambda 750

\begin{tabular}{l|c|c|c}
\hline Интервал длин волн & $250-300$ & $310-400$ & $410-2500$ \\
Погрешность & 0.0095 & 0.0066 & 0.0057
\end{tabular}

Измерение спектральных зависимостей коэффициента отражения ПП проводилось на спектрофотометре Perkin Elmer lambda750. Пределы допускаемой абсолютной погрешности измерения коэффициента отражения приведены в табл. 1.

В результате серии из нескольких процессов, при которых проводилось контролируемое изменение толщин пленок $\mathrm{TiO}_{2}$ и $\mathrm{SiO}_{2}$, была найдена оптимальная комбинация слоев ПП, соответствующая минимальному отражению в требуемом спектральном диапазоне длин волн излучения (300-1800 нм). Толщины пленок $\mathrm{TiO}_{2}$ и $\mathrm{SiO}_{2}$ равны 28 и 99 нм соответственно (см. рис. 1).

\section{4. Расчет фототоков}

Расчет фототоков для каждого субэлемента в СЭ $\mathrm{InGaP} / \mathrm{GaAs} / \mathrm{Ge}$ основывался как на результатах моделирования, так и на экспериментальных данных: в расчете использовались данные смоделированных спектров отражения как для двухслойного ПП $\mathrm{TiO}_{2} / \mathrm{SiO}_{2}$, так и для трехслойного ПП $\mathrm{TiO}_{2} / \mathrm{Si}_{3} \mathrm{~N}_{4} / \mathrm{SiO}_{2}$, а также экспериментально данные для СЭ с оптимизированным двухслойным ПП $\mathrm{TiO}_{2} / \mathrm{SiO}_{2}$. Расчет проводился для стандартного солнечного спектра AM1.5D $\left(1000 \mathrm{BT} / \mathrm{M}^{2}\right)$ с использованием экспериментально определенной спектральной зависимости внутреннего квантового выхода фотоответа CЭ $\mathrm{InGaP} / \mathrm{GaAs} / \mathrm{Ge}$ (рис. 5) [15].

Формула для расчета значений плотностей фототоков имеет следующий вид:

$$
J_{g}=q \int_{\lambda_{\min }}^{\lambda_{\max }^{\max }} F(\lambda)[1-R(\lambda)] Q_{\mathrm{int}}(\lambda) d \lambda,
$$

где $\lambda_{\max }-\lambda_{\min }$ соответствует границам диапазона спектральной чувствительности соответствующего каскада

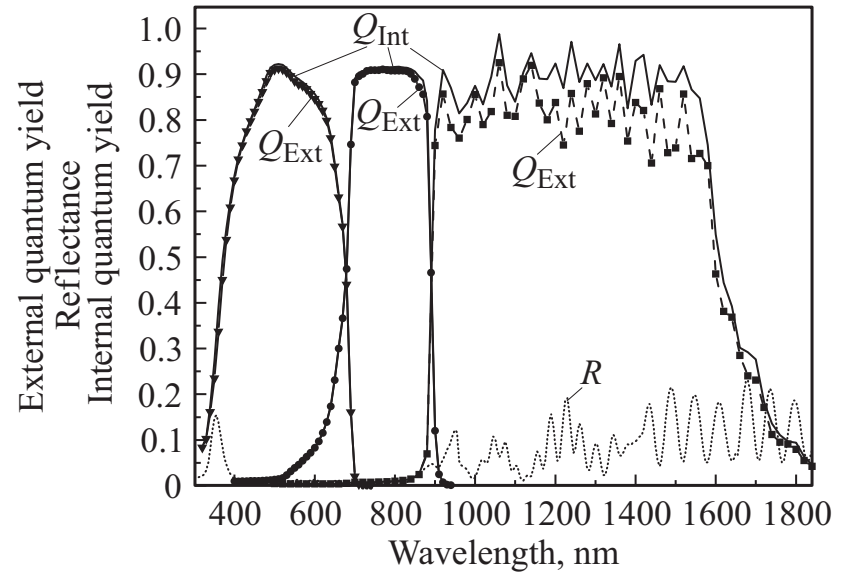

Рис. 5. Экспериментальные спектральные зависимости внешнего $\left(Q_{\text {ext }}\right)$, внутреннего $\left(Q_{\text {int }}\right)$ квантового выхода фотоответа и коэффициента отражения $(R)$ для $\mathrm{C} Э \mathrm{InGaP} / \mathrm{GaAs} / \mathrm{Ge}$.

$(360-680,680-880,880-1800$ нм $), F(\lambda)-$ поток фотонов падающего солнечного излучения, $R(\lambda)$ - коэффициент отражения покрытия, $Q_{\mathrm{int}}(\lambda)-$ внутренний квантовый выход, $q-$ заряд электрона.

В табл. 2 представлены результаты расчета плотностей фототоков отдельных субэлементов $\mathrm{C} Э \mathrm{InGaP} /$ $\mathrm{GaAs} / \mathrm{Ge}$ при использовании смоделированных двухслойного, $\mathrm{TiO}_{2} / \mathrm{SiO}_{2}$, и трехслойного, $\mathrm{TiO}_{2} / \mathrm{Si}_{3} \mathrm{~N}_{4} / \mathrm{SiO}_{2}$, ПП, а также для экспериментально полученного двухслойного ПП $\mathrm{TiO}_{2} / \mathrm{SiO}_{2}$ (условия освещения $1000 \mathrm{BT} / \mathrm{m}^{2}$, AM1.5D). Также в табл. 2 приведен расчет плотностей фототоков при нулевых оптических потерях на отражение.

Из приведенных данных видно, что значения плотностей фототоков для первых двух субэлементов, $\mathrm{InGaP}$ и $\mathrm{GaAs}$, практически не изменяются при переходе от двухслойного, $\mathrm{TiO}_{2} / \mathrm{SiO}_{2}$, к трехслойному, $\mathrm{TiO}_{2} / \mathrm{Si}_{3} \mathrm{~N}_{4} / \mathrm{SiO}_{2}$, ПП. В случае Ge-субэлемента переход к трехслойному ПП дает выигрыш по фототоку на $2.3 \mathrm{MA} / \mathrm{cm}^{2}$. Такая добавка, очевидно, обеспечит небольшое увеличение коэффициента заполнения вольт-амперной характеристики [16], что в свою очередь даст увеличение общей полезной мощности, выдаваемой трехпереходным СЭ $\mathrm{InGaP} / \mathrm{GaAs} / \mathrm{Ge}$ с трехслойным ПП $\mathrm{TiO}_{2} / \mathrm{Si}_{3} \mathrm{~N}_{4} / \mathrm{SiO}_{2}$.

Таблица 2. Рассчитанные значения плотностей фототоков (в мА/см ${ }^{2}$ ) отдельных субэлементов для экспериментально оптимизированного и смоделированных просветляющих покрытий (1000 Bт/м², AM1.5D)

\begin{tabular}{c|c|c|c|c}
\hline \multirow{2}{*}{$\begin{array}{c}\text { Материал } \\
\text { субэлемента }\end{array}$} & Эксперимент & \multicolumn{3}{|c}{ Моделирование } \\
\cline { 2 - 5 } & $\mathrm{TiO}_{2} / \mathrm{SiO}_{2}$ & $\mathrm{TiO}_{2} / \mathrm{SiO}_{2}$ & $\mathrm{TiO}_{2} / \mathrm{Si}_{3} \mathrm{~N}_{4} / \mathrm{SiO}_{2}$ & $\begin{array}{c}\text { Идеальное ПП } \\
(R=0)\end{array}$ \\
\hline $\mathrm{InGaP}$ & 14.54 & 14.50 & 14.53 & 13.74 \\
$\mathrm{GaAs}$ & 13.65 & 13.54 & 21.89 & 14.10 \\
$\mathrm{Ge}$ & 19.52 & 19.58 & & 23.20
\end{tabular}


Таблица 3. Погрешности рассчитанных значений плотностей фототоков для экспериментального ПП $\mathrm{TiO}_{2} / \mathrm{SiO}_{2}$

\begin{tabular}{c|c|c}
\hline $\begin{array}{c}\text { Материал } \\
\text { субэлемента }\end{array}$ & $\begin{array}{c}\text { Абсолютная } \\
\text { погрешность } \\
\Delta J_{g}, \mathrm{MA} / \mathrm{cm}^{2}\end{array}$ & $\begin{array}{c}\text { Относительная } \\
\text { погрешность } \\
\left(\Delta J_{g} / J_{g}\right) \cdot 100, \%\end{array}$ \\
\hline $\mathrm{InGaP}$ & 0.14 & 0.96 \\
$\mathrm{GaAs}$ & 0.13 & 0.97 \\
$\mathrm{Ge}$ & 0.22 & 1.12
\end{tabular}

Оценки абсолютной и относительной погрешностей для значений плотностей токов при использовании экспериментального ПП $\mathrm{TiO}_{2} / \mathrm{SiO}_{2}$ представлены в табл. 3.

\section{5. Заключение}

Проведено моделирование двух- и трехслойных ПП для трехпереходных $\mathrm{C} Э \mathrm{InGaP} / \mathrm{GaAs} / \mathrm{Ge}$. Разработаны режимы осаждения слоев ПП методом реактивного магнетронного распыления и экспериментально получено оптимальное двухслойное ПП $\mathrm{TiO}_{2} / \mathrm{SiO}_{2}$. Произведены оценки значений плотностей фототоков для субэлементов, входящих в состав $\mathrm{C} Э \mathrm{InGaP} / \mathrm{GaAs} / \mathrm{Ge}$, при использовании двухслойного, $\mathrm{TiO}_{2} / \mathrm{SiO}_{2}$, и трехслойного, $\mathrm{TiO}_{2} / \mathrm{Si}_{3} \mathrm{~N}_{4} / \mathrm{SiO}_{2}$, ПП. Показано, что трехслойное ПП $\mathrm{TiO}_{2} / \mathrm{Si}_{3} \mathrm{~N}_{4} / \mathrm{SiO}_{2}$ дает ощутимый прирост по величине плотности фототока в $\mathrm{Ge}-$ субэлементе, что в результате должно положительно отразиться на регистрируемых значениях кпд СЭ $\mathrm{InGaP} / \mathrm{GaAs} / \mathrm{Ge}$.

Работа выполнена при поддержке Российского научного фонда, соглашение № 14-29-00178.

\section{Список литературы}

[1] Minwoo Nam, Kangho Kim, Jaejin Lee, Kee-Keun Lee, Sang Sik Yang. Solar Energy, 91, 374 (2013).

[2] M. Stan, D. Aiken, B. Cho, A. Cornfeld, V. Ley, P. Patel, P. Sharps, T. Varghese. J. Cryst. Growth, 312 (8), 1370 (2010).

[3] P. Patel, D. Aiken, D. Chumney, A. Cornfeld, Y. Lin, C. Mackos, J. McCarty, N. Miller, P. Sharps, M. Stan. Proc. 38th IEEE PVSC (New Mexico, USA, 2012) p. 1.

[4] P. Patel, D. Aiken, A. Boca, B. Cho, D. Chumney, M.B. Clevenger, A. Cornfeld, N. Fatemi, Y. Lin, J. McCarty, F. Newman, P. Sharps, J. Spann, M. Stan, J. Steinfeldt, C. Strautin, T. Varghese. IEEE J. Photovoltaics, 2 (3), 377 (2012).

[5] D. Friedman. Curr. Opin. Solid State Mater. Sci., 14 (6), 131 (2010).

[6] R. King, D. Law, K. Edmondson, C. Fetzer, G. Kinsey, H. Yoon, D. Krut, J. Ermner, R. Sherif, N. Karam. Adv. OptoElectron., 2007, Article ID 29523 (2007). DOI: $10.1155 / 2007 / 29523$

[7] M. Born, E. Wolf. Principles of optics (Oxford-LondonEdinburgh-N. Y.-Paris-Frankfurt, Pergamon Press, 1968) p. 66.

[8] J.R. Devore. J. Opt. Soc. Am., 41 (6), 416 (1951).
[9] H.R. Philipp. J. Electrochem. Soc., 120 (2), 295 (1973).

[10] I.H. Malitson. J. Opt. Soc. Am., 55 (10), 1205 (1965).

[11] D.J. Aiken. Sol. Energy Mater. Sol. Cells, 64, 393 (2000).

[12] G.R. Moriarty, J.T. Beechinor, M. Murtagh, P.V. Kelly, G.M. Crean, S.W. Bland. Thin Solid Films, 364 (1-2), 244 (2000).

[13] J.B. Theeten, D.E. Aspnes, R.P.H. Chang. J. Appl. Phys., 49 (12), 6097 (1978).

[14] D.E. Aspnes, A.A. Studna. Phys. Rev. B, 27 (2), 985 (1983).

[15] S.A. Mintairov, V.M. Andreev, V.M. Emelyanov, N.A. Kalyuzhnyy, N.Kh. Timoshina, M.Z. Shvarts, V.M. Lantratov. Semiconductors, 44 (8), 1084 (2010).

[16] H. Yoon, M. Haddad, S. Mesropian, J. Yen, K. Edmondson, D. Law, R.R. King, D. Bhusari, A. Boca, N. Karam. Proc. 33rd IEEE PVSC (San Diego, California, USA, 2008) p. 82.

Редактор Л.В. Шаронова

\section{Influence of double-layer and triple-layer anti-reflection coatings on formation of the photocurrents in multi-junction $A^{\prime \prime \prime} B^{V}$ solar cells}

\author{
S. Musalinov ${ }^{1}$, A. Anzulevich ${ }^{1}$, I. Bychkov ${ }^{1}$, \\ A. Gudovskikh ${ }^{2}$, M. Shvarts ${ }^{3}$ \\ ${ }^{1}$ Chelyabinsk State University, \\ 454021 Chelyabinsk, Russia \\ 2 St. Petersburg Academic University, \\ 194021 St. Petersburg, Russia \\ 3 loffe Institute, \\ 194021 St. Petersburg, Russia
}

\begin{abstract}
The results of simulation by using transfer matrix method of $\mathrm{TiO}_{2} / \mathrm{SiO}_{2}$ double-layer and $\mathrm{TiO}_{2} / \mathrm{Si}_{3} \mathrm{~N}_{4} / \mathrm{SiO}_{2}$ triplelayer anti-reflection coatings for multi-junction heterostructural InGaP/GaAs/Ge solar cells are presented. $\mathrm{TiO}_{2} / \mathrm{SiO}_{2}$ double-layer anti-reflection coating has been experimentally developed and optimized. By using experimental spectral dependences of measured external quantum yield of $\mathrm{InGaP} / \mathrm{GaAs} / \mathrm{Ge}$ heterostructure solar cell and optical characteristics of anti-reflection coatings, which were got during simulation, values of photogenerated current densities of each subcell in $\mathrm{InGaP} / \mathrm{GaAs} / \mathrm{Ge}$ solar cell under AM1.5D $\left(1000 \mathrm{~W} / \mathrm{m}^{2}\right)$ and lack of losses on reflection conditions have been calculated. Within simulation it is shown that optimized $\mathrm{TiO}_{2} / \mathrm{Si}_{3} \mathrm{~N}_{4} / \mathrm{SiO}_{2}$ triple-layer anti-reflection coating gives a gain in magnitude of generation current density on $2.3 \mathrm{~mA} / \mathrm{cm}^{2}$ for $\mathrm{Ge}$ cascade under AM1.5D in comparison with $\mathrm{TiO}_{2} / \mathrm{SiO}_{2}$ doublelayer anti-reflection coating under the same condition, providing thereby growth of fill factor of current-voltage curve and output electric power.
\end{abstract}

